\title{
Quantization of entropy in a quasi-two-dimensional electron gas
}

\author{
A. A. Varlamov, ${ }^{1}$ A. V. Kavokin, ${ }^{2}$ and Y. M. Galperin ${ }^{3,4}$ \\ ${ }^{1}$ CNR-SPIN, Viale del Politecnico 1, I-00133, Rome, Italy \\ ${ }^{2}$ CNR-SPIN, Viale del Politecnico 1, I-00133, Rome, Italy and Physics and Astronomy School, \\ University of Southampton, Southampton, SO171BJ, United Kingdom \\ ${ }^{3}$ Department of Physics, Oslo University, P.O. Box 1048, Blindern, 0316 Oslo, Norway \\ ${ }^{4}$ A. F. Ioffe Physico-Technical Institute RAS, 194021 St. Petersburg, Russian Federation
}

(Received 27 December 2015; revised manuscript received 13 March 2016; published 4 April 2016)

\begin{abstract}
We demonstrate that the entropy per electron of a two-dimensional electron gas (2DEG) exhibits quantized peaks at resonances between the chemical potential and electron levels of size quantization. In the limit of no scattering, the peaks depend only on the subband quantization number and are independent of material parameters, shape of the confining potential, electron effective mass, and temperature. The quantization of entropy per electron is a signature of a Lifshitz phase transition in a 2DEG. In the presence of stationary disorder, the magnitude of peaks decreases. Its deviation from the quantized values is a direct measure of the disorder induced smearing of the electronic density of states.
\end{abstract}

DOI: 10.1103/PhysRevB.93.155404

Introduction. Low-dimensional electronic devices are important building blocks for quantum electronics. This is one of the reasons for great interest in these systems. Another reason is that size quantization of the electronic states in low-dimensional systems leads to quantization of their thermal and transport properties. The most famous are the integer [1] and fractional [2] quantum Hall effect in two-dimensional electron gas (2DEG) and conductance quantization of quasione-dimensional channels [3].

In this paper, we address the major thermodynamic quantity - entropy — of a quasi-two-dimensional electron gas. An elegant way to measure directly the entropy per electron, $s \equiv(\partial S / \partial n)_{T}$, was recently demonstrated [4]. Here $T$ is temperature. We will show that the quantization of the energy spectrum of quasi-2DEG into subbands leads to a very specific quantization of the entropy: $s$ exhibits sharp maxima as the chemical potential $\mu$ passes through the bottoms of size quantization subbands $\left(E_{i}\right)$. The value of the entropy per electron at the intersection of the chemical potential with the $N$ th size quantization level depends only on the number of the maximum $N$ :

$$
\left.s\right|_{\mu=E_{n}} \equiv\left(\frac{\partial S}{\partial n}\right)_{T, \mu=E_{n}}=\frac{\ln 2}{N-1 / 2} .
$$

In the absence of scattering this result is independent of the shape of the transversal potential that confines $2 \mathrm{DEG}$ and of the material parameters including the electron effective mass and dielectric constant.

The universality of the above quantization rule can be broken both by disorder and by electron-electron interactions. Using a simple model of Lorentzian smearing of the electronic spectrum we show that it leads to the relative correction of $\sim \hbar / T \tau$, where $\tau$ is the electron lifetime. For the case of a single-band 2DEG the role of the electron-electron interaction in the entropy per electron was investigated in Ref. [4], see references therein for a review. We believe that Eq. (1) can be used as a benchmark allowing us to judge the importance of the disorder and interactions. In this paper we report on the analytical dependence of the entropy per electron on the chemical potential accounting for the smeared density of states of 2DEG at the electron quantization levels. We reveal the quantization of entropy per electron at resonances of the chemical potential and electron quantization levels and discuss the accuracy of the obtained expression for the quantized entropy in the presence of disorder and electron-electron interactions.

General expressions. In the absence of scattering, the density of electronic states (DOS) in a noninteracting 2DEG has a staircaselike shape [5],

$$
g(\mu)=\frac{m^{*}}{\pi \hbar^{2}} \sum_{i=1}^{\infty} \theta\left(\mu-E_{i}\right), x
$$

with $m^{*}$ being the electron effective mass and $\theta(x)$ being the Heaviside theta function. Elastic scattering of electrons on defects and impurities that is necessarily present in realistic systems leads to the smearing of the steps of the density of states. A simple way to account for this smearing is to introduce a finite lifetime $\tau$ of an electron. That results in the replacement of a Dirac delta function by a Lorentzian in the derivative of the density of states: $\theta^{\prime}(E)=\delta(E) \rightarrow \hbar \tau^{-1} / \pi\left(E^{2}+\hbar^{2} \tau^{-2}\right)$. Integration of the latter expression leads to the replacement $\theta(E) \rightarrow \tilde{\theta}(E)$, where

$$
\tilde{\theta}(E)=\frac{1}{2}+\frac{1}{\pi} \arctan \left(\frac{E \tau}{\hbar}\right) .
$$

We will focus on a case where $T \gg \hbar / \tau$, that corresponds to a relatively clean sample. At the same time, temperatures are supposed to be not too high, $T \ll \Delta_{N j}=\left|E_{N}-E_{j}\right|, \forall j \neq N$. In addition, we assume that the transport is adiabatic [6], i.e., there are no elastic interband transitions due to backscattering.

To find the entropy per electron $s$ we use the Maxwell relation

$$
s=\left(\frac{\partial S}{\partial n}\right)_{T}=-\left(\frac{\partial \mu}{\partial T}\right)_{n}=\left(\frac{\partial n}{\partial T}\right)_{\mu}\left(\frac{\partial n}{\partial \mu}\right)_{T}^{-1} .
$$

The relationship between the electron concentration $n$, chemical potential $\mu$, and temperature $T$ can be found integrating Eq. (2) over energies with the Fermi-Dirac distribution and 
accounting for the renormalization (3):

$$
n(\mu, T)=\frac{m^{*}}{\pi \hbar^{2}} \sum_{i=1}^{\infty} \int_{0}^{+\infty} \frac{\tilde{\theta}\left(E-E_{i}\right)}{\exp \left(\frac{E-\mu}{T}\right)+1} d E .
$$

Calculating the partial derivatives of the electron concentration over temperature and chemical potential one can express them in the form of sums over the subband levels averaged over energy with temperature and impurities smearing factors:

$$
\begin{gathered}
\left(\frac{\partial n}{\partial T}\right)_{\mu}=\frac{m^{*}}{\pi^{2} \hbar^{2}} \sum_{j=1}^{\infty} \int_{-\infty}^{+\infty} \frac{f_{n}(z)}{\cosh ^{2} z} d z, \\
\left(\frac{\partial n}{\partial \mu}\right)_{T}=\frac{m^{*}}{2 \pi^{2} \hbar^{2}} \sum_{j=1}^{\infty} \int_{-\infty}^{+\infty} \frac{f_{\mu}(z)}{\cosh ^{2} z} d z, \\
f_{n}(z) \equiv z \arctan \left[\left(2 T z+\delta_{N}+\Delta_{N j}\right) \tau\right], \\
f_{\mu}(z) \equiv \pi / 2+\arctan \left[\left(2 T z+\delta_{N}+\Delta_{N j}\right) \tau\right] .
\end{gathered}
$$

Here $\delta_{N}=\mu-E_{N}$ (we assume $\left|\delta_{N}\right| \ll \Delta_{N N \pm 1}$ ). We have also taken into account that $\mu \rightarrow E_{N} \gg T$ and extended the lower limit of integration up to $-\infty$.

Noninteracting $2 D E G$ in the absence of disorder. We start the analysis with the case of a clean material, where one can neglect the smearing of electron states and replace $\arctan z \rightarrow(\pi / 2) \operatorname{sign} z$. In this case the principal contribution to the derivative (6) gives the level closest to the chemical potential:

$$
\left(\frac{\partial n}{\partial T}\right)_{\mu \rightarrow E_{N}}=\frac{m^{*}}{\pi \hbar^{2}}\left[\ln \left(2 \cosh \frac{\delta_{N}}{2 T}\right)-\frac{\delta_{N}}{2 T} \tanh \frac{\delta_{N}}{2 T}\right] .
$$

The contributions of other levels are exponentially small; they turn out to be of the order of $\exp \left(-\Delta_{N, N \pm 1} / T\right)$. In Eq. (7), the lowest $N-1$ levels provide the same universal, independent on chemical potential and temperature, contributions, while the shape of the line is determined by the $N$ th level. We obtain:

$$
\left(\frac{\partial n}{\partial \mu}\right)_{\mu \rightarrow E_{N}}=\frac{m^{*}}{\pi \hbar^{2}}\left(N-\frac{1}{2}\right)+\frac{m^{*}}{2 \pi \hbar^{2}} \tanh \frac{\delta_{N}}{2 T} .
$$

The contributions of the higher levels $(j>N)$ are exponentially small.

Finally, the expression for the entropy per electron Eq. (4), valid for any spectrum of size quantization $E_{j}$, takes the form:

$$
\begin{gathered}
s_{\mu \rightarrow E_{N}}=\frac{\ln \left(2 \cosh \frac{\delta_{N}}{2 T}\right)-\frac{\delta_{N}}{2 T} \tanh \frac{\delta_{N}}{2 T}}{(N-1 / 2)+\frac{1}{2} \tanh \frac{\delta_{N}}{2 T}} \\
=\left\{\begin{array}{cc}
\frac{\left|\delta_{N}\right|}{T} \frac{\exp \left(-\frac{\left|\delta_{N}\right|}{T}\right)}{N-1+\exp \left(-\frac{\left|\delta_{N}\right|}{T}\right)}, & \delta_{N} \ll-T, \\
\frac{\ln 2}{N-1 / 2}, & 0 \leqslant \delta_{N} \ll T, \\
\frac{\delta_{N}}{T N} \exp \left(-\delta_{N} / T\right), & \delta_{N} \gg T .
\end{array}\right.
\end{gathered}
$$

This expression predicts the existence of quantized peaks of the partial entropy $s$ at $\mu=E_{N}$, their magnitudes being dependent only on the subband number. The dependence of $s$ on the chemical potential is schematically shown in Fig. 1, lower panel. The quantized peaks of the entropy per electron correspond to the steps of the density of states shown in the upper panel of the same figure.

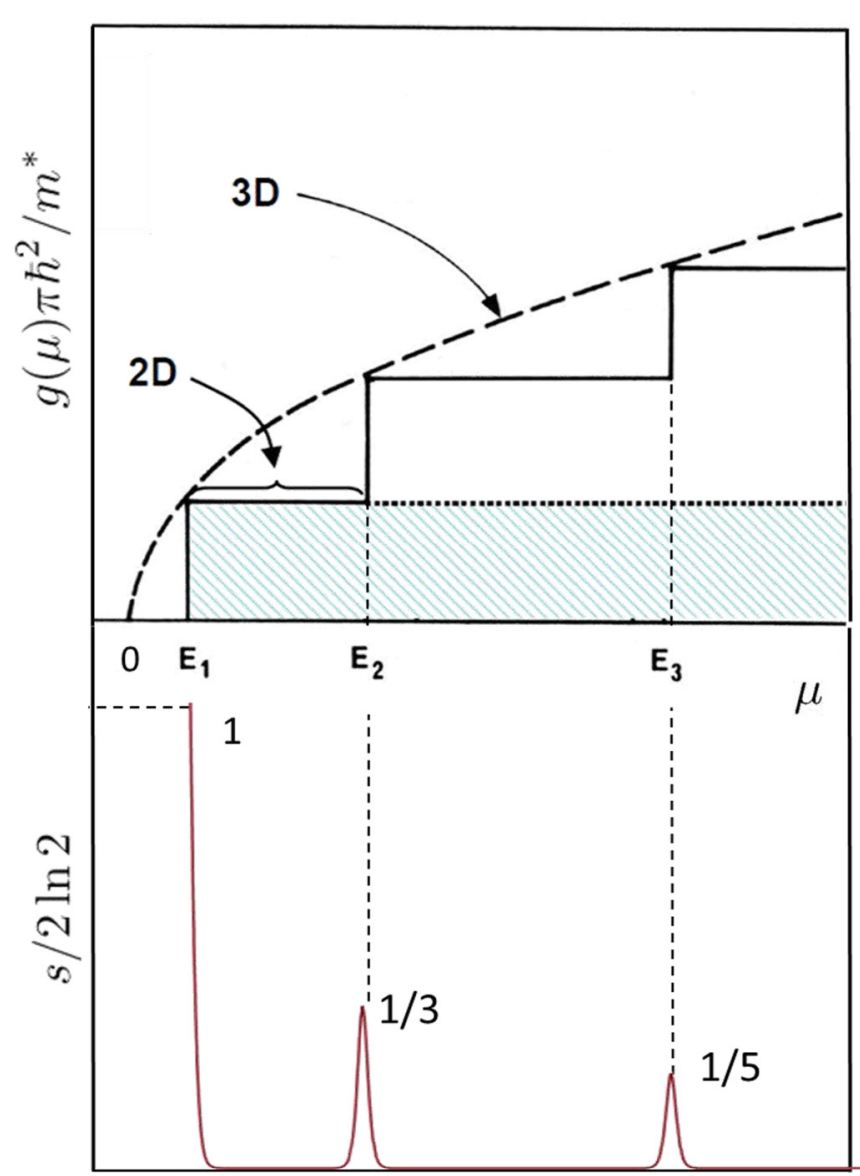

FIG. 1. Schematic representation of the dependencies of the electronic density of states (upper panel) and the entropy per electron (lower panel) as functions of the chemical potential.

The shape of the peaks in Eq. (8) is asymmetric; that corresponds to the steplike changes in the density of electronic states as a function of the chemical potential of the 2DEG.

Effect of scattering. Equations (6) and (7) allow one to estimate the effect of scattering on the heights of the peaks. A straightforward analysis shows that the contribution of the lower subbands is of the order of $\sum_{j=1, j \neq N}^{\infty} \mathcal{O}\left[\left(\Delta_{N j} \tau / \hbar\right)^{-n}\right]$. Here $n=1$ for $(\partial n / \partial \mu)_{T}$ and $n=2$ for $(\partial n / \partial T)_{\mu}$. These sums are cut off at $j_{\max } \sim \hbar / T \tau$. For the equidistant spectrum (parabolic potential), or $E_{N} \sim N^{2 / 3}$ (eigenvalues of the Airy functions, in the case of the linear potential) they give small contributions of the order $(\Delta \tau / \hbar)^{-n}(T / \Delta)^{n-1}(\Delta$ is the characteristics scale of interlevel distances).

The contribution of the $N$ th subband to $(\partial n / \partial \mu)_{T}$ can be calculated exactly leading to the replacement

$$
\tanh \frac{\delta_{N}}{2 T} \rightarrow \operatorname{Re}\left[\tanh \frac{\delta_{N}}{2 T}-i \frac{\hbar}{2 \tau T}\right]
$$

in Eq. (8), i.e., to appearance of the corrections of the order $\mathcal{O}\left[(\hbar / T \tau)^{2}\right]$. Yet, the dominant effect of impurities is due to $(\partial n / \partial T)_{\mu}$. The asymptotic analysis of Eq. (6) shows that the magnitude of the peak in $s$ is suppressed by the elastic 
scattering of electrons as

$$
s_{\mu=E_{N}}=\frac{\ln 2-(\hbar / \pi T \tau)}{N-1 / 2} .
$$

This simple relation allows us to characterize the degree of disorder in a $2 \mathrm{DEG}$.

Discussion. The dependence of the entropy per electron $s$ on the chemical potential can be interpreted in the following way. At low temperatures, the main contribution to the entropy is provided by the electrons having energies in the vicinity of the Fermi level, the width of the 'active' layer being $\sim T$. If the electron DOS is constant within the layer then by adding an electron one does not change the entropy. Hence, the entropy is independent of the chemical potential, $(\partial S / \partial n)_{T} \rightarrow 0$. However, if the bottom of one of the subbands falls into the active layer, the number of 'active' states becomes strongly dependent on the chemical potential. In this case, adding an electron to the system, one changes the number of 'active' states in the vicinity of the Fermi surface. Consequently, the entropy per electron strongly increases. The peaks of the entropy per electron correspond to the resonances of the chemical potential and electron size quantization levels. The further increase of the chemical potential brings the system to the region of the constant density of states, where the entropy per electron vanishes again.

The intersections by the chemical potential of the levels of electron size quantization, $\delta_{N}=0$, can be considered as the points of Lifshitz phase transitions in a 2DEG, where the Fermi surface acquires a new component of topological connectivity. Corresponding anomalies in the thermodynamic and transport characteristics, in particular, thermoelectric coefficient related to the peculiarities of the energy dependence of the electron momentum relaxation time have been studied experimentally and theoretically in Refs. [7,8] and Ref. [9], respectively. Here we present an analytical theory of purely thermodynamic anomalies.

In the asymptotic expression (9) for strongly negative $\delta_{N}$ (but $\left|\delta_{N}\right| \gg T$ ), the term $\exp \left(-\left|\delta_{N}\right| / T\right)$ in the denominator can be neglected for all $N>1$. However, it becomes important for $N=1$. Indeed, at $\mu<E_{1}$ the entropy per electron increases as $\left|\mu-E_{1}\right| / T$ with decreasing $\mu$. This is a manifestation of the crossover from the Fermi distribution to the Boltzmann one when the chemical potential falls into the gap in the spectrum. The region $\mu<E_{1}$ is not shown in Fig. 1 in order to keep the peaks for $N=2,3$ visible.

At $T \rightarrow 0$ (yet $T \gtrsim \hbar / \tau)$ the peaks of $s$ are located at $\mu \rightarrow$ $E_{N}, N>1$, the maximal values being $s_{\max }(N)=\ln 2 /(N-$ $1 / 2)$. At finite $T$ the peaks acquire finite widths of the order of $T$ and shift toward negative values of $\mu-E_{N}$.

The peaks of $s$ for $N=2$ and 3 are shown in Fig. 2; their characteristics are given in Table I. The reason of the peaks' asymmetry is the difference in partial densities of states above and below the chemical potential. The relative difference between the DOSs decreases with increase of $N$, therefore the peaks become more symmetric.

Interestingly, our result for $(\partial \mu / \partial T)_{n} \equiv-s$ at $\mu=E_{1}$ and $\tau \rightarrow \infty$ coincides with the expression for the same derivative obtained in Refs. [10,11] for a two-dimensional superconductor. The quantized dip in this derivative is associated with the step in the density of electronic states which changes from zero

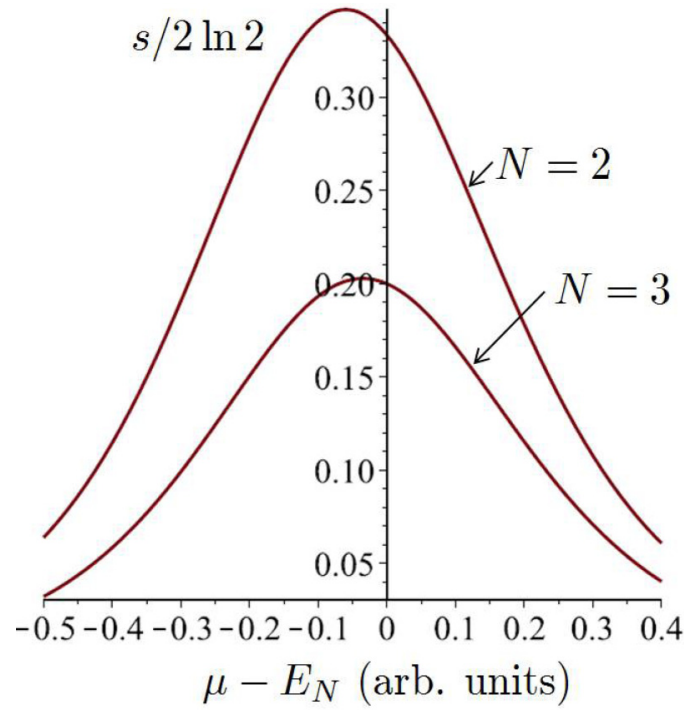

FIG. 2. Dependence $s\left(\mu-E_{N}\right)$ for $N=2,3$.

inside the superconducting gap to $m^{*} / \pi \hbar^{2}$ above the gap. A remarkable fact is that the value of the effective mass $m^{*}$ does not enter the result. Note that the variation of the chemical potential as a function of temperature can be measured by resonant optical transmission spectroscopy of the fundamental absorption edge in modulation doped semiconductor quantum wells, see, e.g., Ref. [12].

Now let us briefly discuss the role of electron-electron (e-e) interactions, which are neglected in the above formalism. Electron-electron interactions become noticeable for the electronic states sufficiently close to the subbands' bottoms. In particular, they can significantly change the compressibility of the electron gas, see, e.g., Ref. [13]. Characterizing the importance of e-e interaction by the parameter $r_{s}$ [14] we conclude that $r_{s} \approx 1$ for the upper filled subband at

$$
\sqrt{\frac{2 \delta_{N}}{m^{*}}} \approx \frac{e^{2}}{\kappa \hbar} \rightarrow \delta_{N} \approx \frac{m^{*}}{2}\left(\frac{e^{2}}{\kappa \hbar}\right)^{2},
$$

with $\kappa$ being a dielectric constant. Putting $m^{*}=0.1 m_{0}$ and $\kappa=10$ we get $\delta_{N} \gtrsim 2 \times 10^{-14} \mathrm{erg}$. If $\delta_{N}$ is less than this value one can expect a Fermi-liquid renormalization of the electron spectrum, in particular, of the effective mass $m^{*}$. Fortunately, $m^{*}$ doesn't enter the expressions for the peaks of $s$. However, additional correction proportional to $\left(\partial m^{*} / \partial n\right)$ can appear in the expression (7) for the thermodynamic DOS. These corrections for $N=1$ are discussed in Ref. [4] and references therein. Another possibility of evidencing the interaction effects is establishing a special regime of a correlated 2D charged plasma [15] also explored in Ref. [4]. Here we do not consider this particular case. In general, comparing the

TABLE I. Peaks in the entropy per electron.

\begin{tabular}{cccc}
\hline \hline$N$ & $\delta_{\max } / 2 T$ & $s_{\max } / 2 \ln 2$ & $\left.s\right|_{\mu=E_{N}} / 2 \ln 2$ \\
\hline 2 & -0.24 & 0.347 & $1 / 3$ \\
3 & -0.14 & 0.203 & $1 / 5$ \\
4 & -0.01 & 0.144 & $1 / 7$ \\
\hline \hline
\end{tabular}


experimental results with the universal expression obtained here (8) allows one to judge on the role of electron-electron correlations in a system under study.

Finally, we would like to emphasize that the peak values of entropy per particle are not quantized, strictly speaking, as one can clearly see from Figure 2 . The peaks are shifted from the topological transition points, and their magnitudes are sensitive to the thermal broadening, elastic scattering, and electron-electron interactions. On the other hand, according to our model, the entropy per particle measured at the 2D Lifshitz transition points is quantized. Its value is independent of any structure parameter: of the effective mass, dielectric constant, shape of the confining potential, temperature. This makes us hope that this quantization rule is universal and quite robust against disorder, interaction effects, and temperature.
In conclusion, we have analytically derived the entropy per electron $(\partial S / \partial n)_{T}$ in a noninteracting quasi-2DEG in the vicinity of electron quantization levels. At the resonances of the chemical potential with the electron quantization levels (2D Lifshitz transition points), $(\partial S / \partial n)_{T}$ appears to be independent of the effective mass, dielectric constant, and other material parameters. The entropy per electron may be directly measured either by temperature modulation [4], or by the optical transmission measurements [12].

A.V. and A.K. acknowledge partial support from the HORIZON 2020 RISE project CoExAn (Grant No. 644076). Y.G. acknowledges partial support from the EU FP7 project INFERNOS (Grant No. 308850).
[1] K. von Klitzing and G. Dorda, and M. Pepper, Phys. Rev. Lett. 45, 494 (1980).

[2] D. C. Tsui, H. L. Stormer, and A. C. Gossard, Phys. Rev. Lett. 48, 1559 (1982).

[3] D. A. Wharam, T. J. Thornton, R. Newbury, M. Pepper, H. Ahmed, J. E. F. Frost, D. G. Hasko, and D. C. Peacock, D. A. Ritchie and G. A. C. Jones, J. Phys. C 21, L209 (1988); B. J. van Wees, H. van Houten, C. W. J. Beenakker, J. G. Williamson, L. P. Kouwenhoven, and D. van der Marel, and C. T. Foxon, Phys. Rev. Lett. 60, 848 (1988).

[4] A. Yu. Kuntsevich, V. M. Pudalov, I. V. Tupikov, and I. S. Burmistrov, Nat. Commun. 6, 7298 (2015).

[5] T. Ando, A. B. Fowler, and F. Stern, Rev. Mod. Phys. 54, 437 (1982).

[6] C. W. J. Beenakker and H. van Houten, in Solid State Physics, edited by H. Ehrenreich and D. Turnbull (Academic Press, Boston, 1991), Vol. 44, pp. 1-228.
[7] N. V. Zavaritskii and Z. D. Kwon, Pis'ma Zh. Eksp. Teor. Fiz. 39, 61 (1984) [JETP Lett. 39, 71 (1984)].

[8] N. V. Zavaritskii and I. M. Suslov, Zh. Eksp. Teor. Fiz. 87, 2152 (1984) [Sov. Phys. JETP 60, 1243 (1984)].

[9] Ya. M. Blanter, A. V. Pantsulaya, and A. A. Varlamov, Phys. Rev. B 45, 6267 (1992).

[10] B. I. Ivlev and G. M. Eliashberg, Pis'ma Zh. Eksp. Teor. Fiz. 13, 464 (1984) [JETP Lett. 13, 333 (1971)].

[11] A. A. Abrikosov, Fundamentals of the Theory of Metals (Elsevier, Amsterdam, 1989), p. 414.

[12] D. Dutton, Phys. Rev. 112, 785 (1958).

[13] J. P. Eisenstein, L. N. Pfeiffer, and K. W. West, Phys. Rev. B 50, 1760 (1994).

[14] G. D. Mahan, Many-Particle Physics (Kluwer Academic/Plenum Publishers, New York, Boston, Dodrecht, London, Moscow, 1981), p. 380.

[15] D. S. Novikov, Phys. Rev. B 79, 235304 (2009). 